\title{
A Class of Homogeneous Cosmological Models III: Asymptotic Behaviour
}

\author{
M. A. H. MaCCALlum \\ Department of Applied Mathematics and Theoretical Physics, University of Cambridge
}

Received August 14, 1970

\begin{abstract}
The behaviour near to and far from an initial singularity in a broad subclass of the models studied in previous papers [1-3] is examined. The influence of the matter on the evolution at these times is discussed. The singularity types for the various models, which are mostly of cigar or oscillatory nature, are found. It is discovered that among these models, only those of the same Bianchi type as a Robertson-Walker model can become "approximately Robertson-Walker" in a sense defined in the paper. Qualitative conclusions concerning black-body isotropy, the Hubble relation, helium abundance and horizon structure are given.
\end{abstract}

\section{Introduction}

This paper is concerned with the asymptotic behaviour of a class of homogeneous cosmological models studied in previous papers [1-3]. These are spacetimes which satisfy Einstein's field equations for a perfect fluid and admit a three-parameter group of motions $G_{3}$ simply-transitive on spacelike sections orthogonal to the fluid flow vector $u^{a}$. The only spacetimes with three-surfaces of homogeneity orthogonal to $u^{a}$ which do not admit a simply-transitive three-parameter group of motions are those of Case I of Kantowski and Sachs [4].

All non-stationary spatially homogeneous cosmological models (including those in which the matter rotates or accelerates) have singularities, provided that the cosmological constant $\Lambda$ does not have a large positive value [5]. (These singularities are unambiguously physical singularities if the energy density of matter $\mu$ is everywhere positive, and its pressure $p$ is not negative, as will be assumed throughout this paper.) For the models considered here one may deduce the existence of the singularity from the $(00)$ field equation, Raychaudhuri's equation. Defining

$$
u_{a ; b}=: \theta_{a b}=: \frac{1}{3} \theta h_{a b}+\sigma_{a b}
$$


where ${ }^{1} \theta_{a b}=\theta_{(a b)} ; \theta_{a b} u^{b}=0 ; \theta=\theta^{a}{ }_{a} ; h_{a b}=g_{a b}+u_{a} u_{b}$ and $2 \sigma^{2}:=\sigma^{a b} \sigma_{a b}$; this equation reads

$$
\theta+\frac{1}{3} \theta^{2}+2 \sigma^{2}+\frac{1}{2} \mu+\frac{3}{2} p=\Lambda,
$$

where ${ }^{\cdot}$ denotes $d / d t$. The interpretation of the quantities appearing here is that $\theta$ is the expansion of a small congruence of neighbouring worldlines and $\sigma_{a b}$ its shear. With $\mu>0, p \geqq 0$ and $\Lambda \leqq 0$, we find that $\theta+\theta^{2} / 3$ $<0$. Thus if, at time $t, \theta>0$, then there must have been a singularity at some finite time $t^{\prime}<t$.

A sufficiently large positive $\Lambda$ can prevent the occurrence of such a singularity. There are plausible physical arguments for discounting this possibility $[6,7]$ and we consider here only those of our models possessing an initial singularity. Physically this singularity represents the big-bang fireball that appears to have occurred in the real universe $[6,7]$.

One may discuss the late stages of evolution in our models by a similar use of the generalised Friedmann equation, which is a first integral of the field and conservation equations. Denoting the Ricci curvature scalar of the three-surfaces of homogeneity by $R^{*}$, this equation is

$$
\frac{1}{3} \theta^{2}=\sigma^{2}+\mu+\Lambda-R^{*} / 2 .
$$

If $\Lambda \geqq 0$ and $\theta>0$ the general behaviour is that the universe expands for all time; a second singularity, in the future, can only occur if $R^{*}>0$ and this can only happen in universes of Bianchi type IX [1]. If $\Lambda<0$ a second singularity must occur. Since the present-day universe appears approximately isotropic and homogeneous (i.e. like a Robertson-Walker universe), so, at some time in the model, must any realistic model universe.

Thus we are motivated to study the behaviour of our models near to and far from an initial singularity. This paper will in fact only discuss a tractable subset of the models, which is nevertheless a broad class, and consists of all models of Class A and those of Class B in which $n=0$. The classification used here, which is a modification of those of Bianchi [8] and Behr [9], depends on the eigenvalues $n_{1}, n_{2}, n_{3}$ of a symmetric tensor $n_{\alpha \beta}$ and the magnitude $a$ of a vector $a^{\beta}$ which together represent the commutators of a basis of generators of the reciprocal group to the group of motions [1]. The basis is chosen so that $a^{\beta}=(a, 0,0)$ : in general $n_{\alpha \beta}$ is chosen to be diagonal, but when $n:=n_{\alpha}^{\alpha}=0$ one makes the more convenient choice that only $q:=n_{23}=0$. The details of the classification are repeated for reference in Table 1.

1 The notation and conventions follow [1]. Coordinates $\left\{t, x^{\nu}\right\}$ can be chosen so that $\left\{x^{v}\right\}$ are comoving, $\{t=$ constant $\}$ are the surfaces of homogeneity and $t$ is the proper time along the world-lines of the matter. Latin indices run from 0 to 3 , Greek from 1 to 3 . The signature is +2 and $u^{a}$ is normalised $\left(u^{a} u_{a}=-1\right)$. 
Table 1. Summary of the classification of groups $G_{3}$ as introduced in [1]. $h:=a^{2} / n_{2} n_{3}$. Case $B b$ is divided into Bbi and Bbii according as $a^{\beta}$ is or is not a shear eigenvector. Case Bbii can only occur when $n_{2} n_{3}+9 a^{2}=0$ (see [1])

\begin{tabular}{|c|c|c|c|c|c|c|c|}
\hline Class & Case & Type & $a$ & $n_{1}$ & $n_{2}$ & $n_{3}$ & Bianchi type \\
\hline \multirow[t]{6}{*}{ A } & $\mathrm{a}$ & I & 0 & 0 & 0 & 0 & I \\
\hline & b & II & 0 & + & 0 & 0 & II \\
\hline & & $\mathrm{VII}_{0}$ & 0 & 0 & + & + & VII \\
\hline & & $\mathrm{VI}_{0}$ & 0 & 0 & + & - & VI \\
\hline & & VIII & 0 & - & + & + & VIII \\
\hline & & IX & 0 & + & + & + & IX \\
\hline \multirow[t]{4}{*}{ B } & $\mathrm{a}$ & V & + & 0 & 0 & 0 & V \\
\hline & b & IV & + & 0 & 0 & + & IV \\
\hline & & $\mathrm{VII}_{h}$ & + & 0 & + & + & VII \\
\hline & & $\mathrm{VI}_{h}$ & + & 0 & + & - & VI $\left(\begin{array}{c}h=-1 \\
h=-1\end{array}\right)$ \\
\hline
\end{tabular}

In Section 2 we set up a modified version of the potential formalism introduced by Misner for types I [10] and IX [11] and extended by Hawking to all Class A models [12]. In Section 3 some concepts required later are introduced and discussed in the context of the simple subclass in which the three-space curvature $R^{*}{ }_{\alpha \beta}$ is isotropic (i.e. the surfaces of homogeneity are spaces of constant curvature). Sections 4-6 discuss the evolution in the less simple cases ${ }^{2}$ and Section 7 provides a discussion of the implications of the results of the earlier sections. As far as the author is aware, the original references have been given wherever the work of this paper is not original.

\section{The Formalism}

A number of authors have given (essentially equivalent) Lagrangian or Hamiltonian forms of the Einstein equations of spatially-homogeneous universes, with varying degrees of generality [9-12, 14-17]. Although these can be quantised $[11,17]$ here we will only be concerned with "classical" results. The essential virtue of the method, which we apply in a form introduced by Misner $[10,11]$, is that the introduction of a potential makes discussion much clearer. The simple forms used here apply only to Class A and Class B $n=0$ spaces. It is not clear whether it can be applied to general Class B models $[12,16,45]$.

${ }^{2}$ While the final draft of the paper was being written it was discovered that a similar analysis has been carried out by K. C. Jacobs and L. P. Hughston (private communication), and also that further papers on particular cases have appeared $[13,43,44]$. 
In Class $\mathrm{A}$ and $n=0$ cases the metric depends on only three timedependent functions $X, Y, Z[1]$. These we parametrise [10] by

$$
\left.\begin{array}{rl}
X Y Z & =: l^{3}=: \exp (-3 \Omega), \\
X & =: l_{1}=: \exp \left(-\Omega+\beta_{1}\right), \\
Y & =: l_{2}=: \exp \left(-\Omega-\frac{\left(\beta_{1}-\sqrt{3} \beta_{2}\right)}{2}\right), \\
Z & =: l_{3}=: \exp \left(-\Omega-\frac{\left(\beta_{1}+\sqrt{3} \beta_{2}\right)}{2}\right) .
\end{array}\right\}
$$

Only those $n=0$ cases which fall in Class B are treated separately from Class A. In these cases the field equations yield a constraint ([1], $6.3 \mathrm{~b})$

$$
\sqrt{3} \beta_{1}=k \beta_{2}
$$

where $k:=q / a$ is constant for a given $\operatorname{model}^{3}$. The freedom of rescaling of the $l_{\alpha}$ is used in Class A to set $N_{1}:=\frac{n_{1} Y Z}{X}, N_{2}:=\frac{n_{2} Z X}{Y}$, and $N_{3}:=\frac{n_{3} X Y}{Z}$ to \pm 1 or 0 as appropriate (see Table 1 ), and in Class B $n=0$ to set $\sqrt{3} \beta_{1}-k \beta_{2}$ to zero, satisfying (2.2). In the latter cases we introduce $\beta$ defined by

$$
-\sqrt{3+k^{2}} \beta:=k \beta_{1}+\sqrt{3} \beta_{2}
$$

which measures distance along the line in the $\boldsymbol{\beta}$-plane to which a given model is constrained.

We define

$$
V_{1}:=-\frac{2}{3}\left(R^{*}-\delta_{I X}^{T} R_{0}^{*}\right) e^{-2 \Omega}
$$

where $R^{*}$ is the three-space curvature, $R_{0}^{*}=3 / 2 e^{2 \Omega}$ is its value in type IX if $\boldsymbol{\beta}=0$, and $T$ is the group type. $V_{1}$ is considered as a function on the $\boldsymbol{\beta}$-plane only, although it depends implicitly on $t$. Explicitly

Class A:

$$
\begin{aligned}
V_{1}= & \frac{1}{3}\left[N_{1}^{2} e^{4 \beta_{1}}+\left(N_{2} e^{\sqrt{3} \beta_{2}}-N_{3} e^{-\sqrt{3} \beta_{2}}\right)^{2} e^{-2 \beta_{1}}\right. \\
& \left.-2 N_{1} e^{\beta_{1}}\left(N_{2} e^{\sqrt{3} \beta_{2}}+N_{3} e^{-\sqrt{3} \beta_{2}}\right)\right] .
\end{aligned}
$$

Class B $(n=0): \quad V_{1}=\frac{4}{3} a_{0}^{2}\left(3+k^{2}\right) \exp \left(\frac{2 k \beta}{\sqrt{3+\mathrm{k}^{2}}}\right)$

${ }^{3}$ When $k^{2}=1$ one has cases of Bianchi type III which in the terminology of Ellis [18] are locally rotationally symmetric (L.R.S.) and have been investigated by Kantowski and Sachs $\left[4\right.$, cf. 1]. When $k^{2}=9$ one may have the special case Bbii in which $a^{\gamma}$ is not a shear eigenvector. Taking $k=-3$ one then has $\sigma_{12}=b / Y^{2} Z \neq 0$ where $b$ is constant [1]. Note incidentally that the term $\frac{+2 b^{2}}{Y^{4} Z^{2}}$ in $[1](6.7 \mathrm{~b})$ should read $-b^{2} / Y^{4} Z^{2}$. 
where $a_{0}=a X$. To treat case Bbii we introduce

$$
V_{2}(\boldsymbol{\beta})=\frac{4}{3} \sigma_{12}^{2} e^{-6 \Omega}=\frac{4}{3} b^{2} e^{2 \sqrt{3} \beta}
$$

which vanishes except in case Bbii ${ }^{3}$. Finally we define

$$
\begin{gathered}
V(\boldsymbol{\beta}):=V_{1}+V_{2} e^{4 \Omega}, \\
\Phi:=\left(\beta_{1}^{\cdot 2}+\beta_{2}^{\cdot 2}\right) e^{-6 \Omega}+V(\boldsymbol{\beta}) e^{-4 \Omega}=\frac{2}{3} e^{-6 \Omega}\left(2 \sigma^{2}-R^{*}\right) .
\end{gathered}
$$

Essentially $V$ acts as a potential for the $\boldsymbol{\beta}$-motion, while $\Phi$ is effectively Misner's Hamiltonian in the vacuum case ${ }^{4} . V, V_{1}, V_{2}$ and $\Phi$ are nonnegative. Contours of the potential $V$ are shown in Figs. 1-5, for Class A cases except those of Type I where $V \equiv 0$, and Figs. 7-9 for Class B $n=0$ cases. Note that only in Type IX may $R^{*}$ take positive as well as negative values and that then $R^{*}=0 \Leftrightarrow V_{1}=1$. An L.R.S. model ${ }^{3}$ lies on one of the lines $\Theta=0, \Theta= \pm \pi / 3$ where $\Theta$ is a polar angle measured from the $\beta_{1}$-axis.

The remaining Einstein equations are simply

$$
4 \Omega^{\cdot 2}=\Phi e^{6 \Omega}+\mu+\Lambda-\delta_{I X}^{T} e^{2 \Omega}
$$

where $T$ is the group type, and, for each of $\beta_{1}, \beta_{2}$ in Class $\mathrm{A}$, and for $\beta$ in Class $\mathrm{B} n=0$,

$$
2\left(e^{-3 \Omega} \beta^{\cdot}\right)^{\cdot}=-e^{-\Omega} \frac{\partial V}{\partial \beta} .
$$

By (2.9), or directly from ([1], 3.9) which is due to Raychaudhuri,

$$
\Phi^{\prime}=-4 e^{-4 \Omega} V_{1}(\boldsymbol{\beta})
$$

where ' denotes $d / d \Omega$. If $p=p(\mu)$, which is no restriction if $\mu$ does not take the same value twice, we may introduce the thermodynamic variable $w$ (Taub's $\sigma$, see [46]) by

$$
\frac{d w}{w}=\frac{d \mu}{\mu+p} .
$$

The conservation equations $T^{a b}{ }_{; b}=0$ then read simply

$$
w e^{-3 \Omega}=\text { constant } .
$$

It is possible (cf. forthcoming work by Jacobs and Hughston) to formally introduce a potential for the Class $\mathrm{B}(n=0)$ cases valid everywhere in the $\boldsymbol{\beta}$-plane. The peculiar features of these cases (as compared to those of Class A) which will be discovered in subsequent sections may

${ }^{4}$ Matter terms may be added when the matter obeys a Lagrangian description: see e.g. $[10,16,19,46]$. 


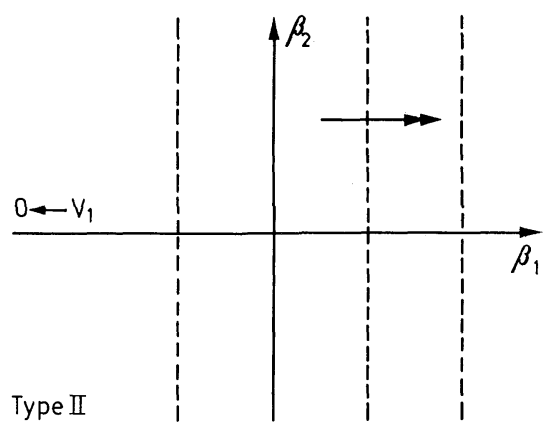

Fig. 1

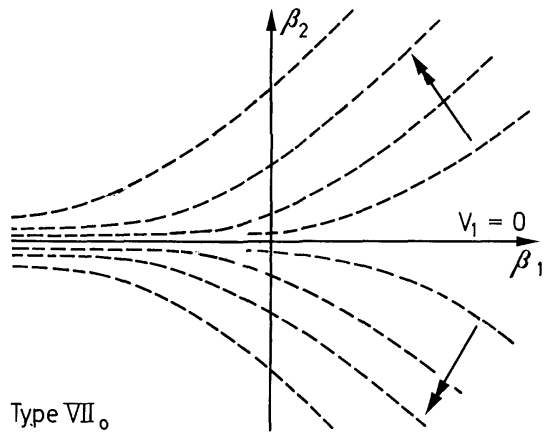

Fig. 3

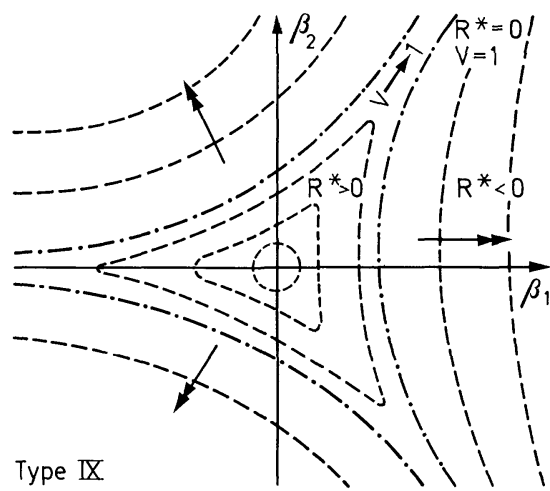

Fig. 5

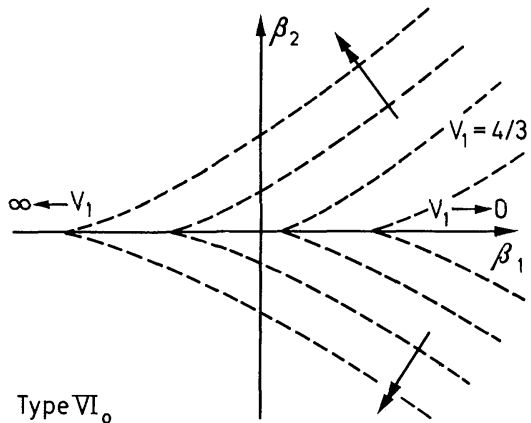

Fig. 2

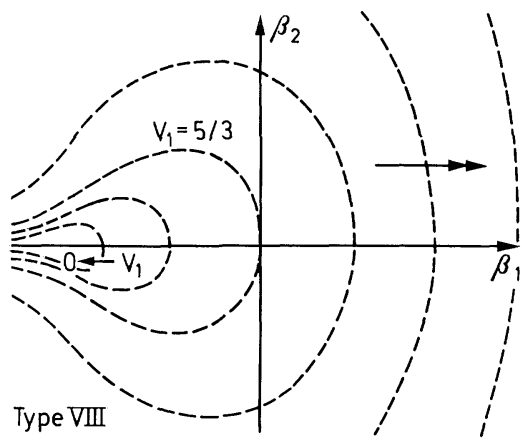

Fig. 4

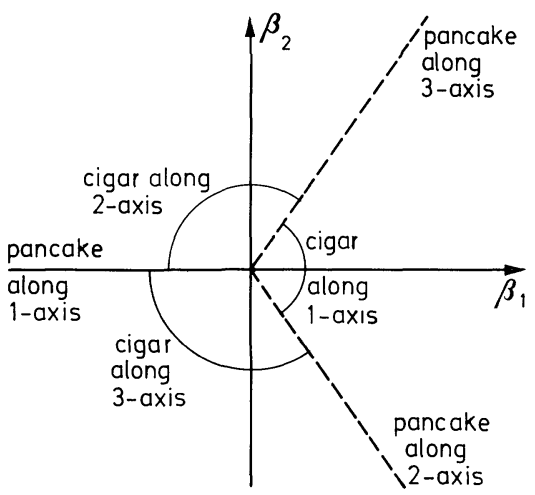

Fig. 6

Figs. 1-5. Contours of $V_{1}(\beta)$ in the various Class A cases. Key: --- contour of $V_{1}$, $\longrightarrow$ direction of exponential increase of $V_{1}$. Certain values and asymptotes of $V_{1}$ are indicated

Fig. 6. Singularity types when system escapes to infinity obeying (3.2) or (4.3) 


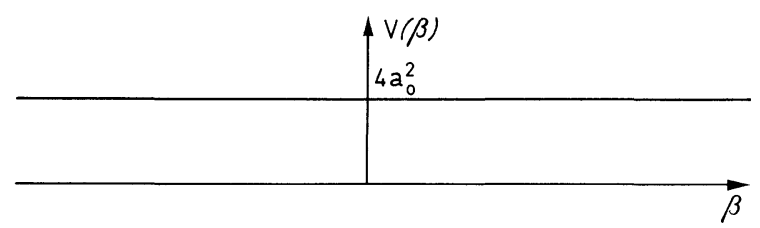

Fig. 7. Case $\mathrm{Ba}$ (Bianchi type $V ; k=0$ ). The potential $V(\beta)$ of $(2.5)$

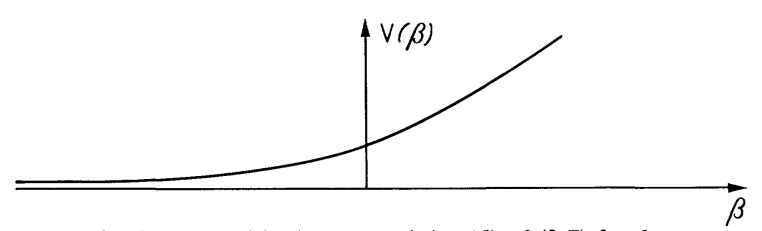

Fig. 8. Case Bbi: the potential $V(\beta)$ of $(2.7)$ for $k>0$

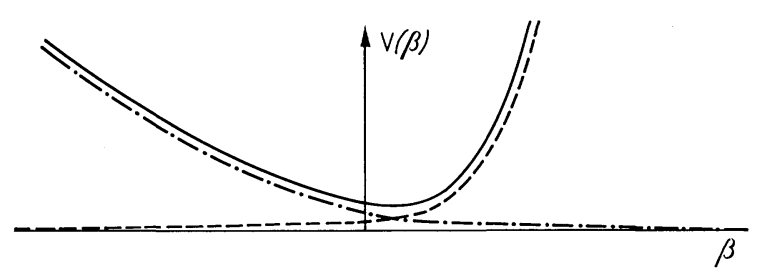

Fig. 9. Case Bbii; $k=-3$ : the potential $V=V_{1}+V_{2} e^{4 \Omega}$ for some arbitrary $\Omega$. --- is $V_{2}$; $-\cdot-V_{1} ;-V$

then be regarded as due to the constraint (2.2) introducing qualitative changes in the dynamics by restricting the system to a fixed line in the $\beta$-plane.

It should be noted that the time $t$ may easily be eliminated from the equations in favour of a new independent variable $\tau$ (defined e.g. by $\tau=\Omega$ or by $d \tau=e^{+3 \Omega} d t$ ). We frequently use $\tau=\Omega$. Whether the possibility has deep significance [20] or not, it "regularises" the equation [16].

(2.9) has the interesting interpretation that the evolution of the extrinsic curvature (second fundamental form) of the three-surfaces of homogeneity is driven, through Einstein's field equations, by the intrinsic curvature in such a way as to increase the latter (i.e. in general make it less negative, so that the actual magnitude decreases).

There are three main advantages of the formalism introduced above. First one is able to use the methods of classical Hamiltonian mechanics (see e.g. [21]). Secondly one can obtain an intuitive picture of the evolution of the models considered. For example, one expects that a type II model must come in from large negative $\beta_{1}$ and rebound out again. Such expectations are largely fulfilled, as we shall see. Thirdly one may treat all the cases, or suitably chosen subsets, simultaneously. 
The remainder of the paper is essentially a study of the solutions of the system of differential equations given by $(2.4-2.10)$ for each type, in the regions of large and small $\Omega$.

\section{The Cases where $R_{\alpha \beta}^{*}$ is Isotropic}

Before starting the analysis it is useful to define some terms, descriptive of various possible behaviours near the singularity, which appear to have been introduced by Thorne [22] and are now in common use. They relate to the asymptotic behaviour, as we go towards the singularity, of a fluid element which at some given finite time is spherical. One considers the lengths of this element in the principal shear directions: if all three tend to zero the singularity is called a "point" singularity ${ }^{5}$; if two tend to zero and one to a finite number, it is called a "barrel" singularity; if two tend to zero and one to infinity, a "cigar" singularity; and if one tends to zero and two to finite numbers, a "pancake" singularity.

It is in general the behaviour near the singularity that governs the existence of particle horizons [23]. In our models we shall use the following definitions ${ }^{6}$ : consider an observer at time $t$ and the set of fluid elements in $\{t=$ constant $\}$ whose past world-lines intersect the observer's past light cone (i.e. which he can see); if this set has a topologically spheical boundary, the boundary is called a particle horizon; if the boundary is cylindrical or toroidal we say the particle horizon has been removed in one direction; and so on. The occurrence of removal of particle horizons depends on the rate of evolution of the models and the topology of the space sections, the latter becoming espectially important in removing horizons at late stages, as happens, for example, in the well-known Lemaître universes.

One would like to require that every observed large-scale property of the universe (e.g. temperature and isotropy of background radiation, helium abundance) can be duplicated at a particular time in the model. However one would need to make a detailed numerical calculation for every case, like those in Refs. [10,12, 22, 25-28], in order to determine whether this was satisfied. Instead we shall frame two weaker criteria we should like the model to satisfy if it expands indefinitely, which indicate whether or not it can be like a Robertson-Walker universe. A weak sense of "becoming approximately Robertson-Walker" is that as $l \rightarrow \infty, \sigma / \theta \rightarrow 0$. This would insure that at sufficiently late stages of the evolution there

${ }^{5}$ This does not generally mean it should be considered as topologically a point. Indeed in the models of this paper the world-line of every particle passes through the singularity at the same cosmic time $t_{\mathrm{s}}$ (which is taken as the time origin) and so the singularity should be considered as topologically a three-surface.

${ }^{6}$ Following Rindler [23] rather than Penrose [24]. 
was no observable deviation from isotropy of the Hubble law. A stronger sense of "becoming approximately Robertson-Walker" is that $\boldsymbol{\beta}$ tends to some finite value as $l \rightarrow \infty$, i.e. $\int_{t_{0}}^{\infty} \sigma_{a b} d t$ is finite for $t_{0}>0$. This stronger condition would ensure that the overall distortion since the decoupling time of the background microwave radiation was finite, and hence there would be a limit on the anisotropy of the observed temperature of this radiation. Neither of these conditions is sufficient to ensure that a model is realistic, nor are they rigorously necessary (in that suitable limits may hold for $\sigma, \beta$ at a given time but not for all later times, cf. [4, 22]). However they clearly offer the best criteria one can provide for rejecting models, other than detailed numerical calculation.

Models which collapse to a second singularity (or asymptotically approach some finite $l$ ) do not allow use of these definitions, for if one considers the initial value problem on a surface near the maximum of $l$ it is clearly possible to find some range of initial data, however restricted, that would enable one to reproduce the observations to the same accuracy as Robertson-Walker models. Here the need for numerical calculations cannot be evaded. Therefore this paper attempts to discuss whether or not a model collapses again, and only if there is no such collapse whether or not the criteria of "becoming approximately Robertson-Walker" outlined above are satisfied.

These definitions having been given, they will be discussed first in the context of the models in which $R^{*}{ }_{\alpha \beta}$ is isotropic. Such models are either Robertson-Walker models of Bianchi types I, V or IX, or anisotropic $(\sigma \neq 0)$ models of Bianchi types I and $\mathrm{V}^{7}$. As the former have been extensively studied by many authors, we remark here only that the isotropy implies that there is a point singularity and that the evolution near the singularity is governed by the matter content. Henceforth this paper treats only models in which $\sigma$ 丰 0 .

Using the results of $[1]$ we have that ${ }^{8}$

$$
\left.\begin{array}{rl}
l_{v} & =l \exp \left(\frac{2 \Sigma}{\sqrt{3}} \sin \alpha_{\gamma} \int \frac{d t}{l^{3}}\right), \\
\alpha_{\nu} & =\alpha+(v-1) \frac{2 \pi}{3}, \\
l \mu^{-} & +3 l(\mu+p)=0, \\
3 l^{2} & =\Sigma^{2} l^{-4}+\mu l^{2}+\Lambda l^{2}+10 E,
\end{array}\right\}
$$

${ }^{7}$ Which if L.R.S. also admit groups of types $\mathrm{VII}_{0}$ and $\mathrm{VII}_{h}$ respectively [1].

${ }^{8}$ In these equations we could use the alternative parameter $\beta_{v}=\frac{2 \pi}{3}-\alpha_{v}$, which is equivalent to changing the sign of the exponential in $l_{v}$ and renumbering (cf. [28]). 
where $E \geqq 0,0 \leqq \alpha<\frac{2 \pi}{3}, \Sigma>0 . \Sigma, E, \alpha$ and $\Lambda$ are constants and $E>0 \Rightarrow \alpha=0$. In terms of the formalism of Section 2 we find that $\beta_{1} e^{-3 \Omega}, \beta_{2} e^{-3 \Omega}$ are constants so the system moves rectilinearly in the $\beta$ plane and $3 \Phi=4\left(\Sigma^{2}+10 E l^{4}\right)$.

First let us consider the case where $\mu>p \geqq 0$ for every component of the fluid ${ }^{9}$. (We shall not here need to specify the equation of state more precisely.) Then at small $l$ (i.e. near the singularity) the matter content is dynamically negligible and

$$
\left.\begin{array}{rl}
3 l^{2} & \simeq \Sigma^{2} l^{-4} \Rightarrow l^{3} \bumpeq \sqrt{3} \Sigma t \\
l_{v} & \simeq\left(3 \Sigma^{2}\right)^{\frac{1}{6}} t^{\frac{1}{3}\left(1+2 \sin \alpha_{\gamma}\right)} .
\end{array}\right\}
$$

Dynamically these are just the vacuum solutions [29, 30]. A cigar singularity occurs unless $\alpha=\pi / 2$. If $\alpha=\pi / 2$ (which implies the space is L.R.S.) a pancake singularity occurs, but among the spaces considered here this is only possible in Bianchi type I. In case Ba (Bianchi type V) we note that the distinguished axis of the cigar singularity is not that picked out by the vector $a^{\beta}$ (which with the present conventions lies along the 1 axis). In the formalism of Section 2, when $l \rightarrow 0(\Omega \rightarrow \infty)$ in these models, $\Phi \simeq \frac{4}{3} \Sigma^{2}, 4 \Omega^{2} \simeq \Phi e^{6 \Omega}$, and $\beta_{1}^{\prime 2}+\beta_{2}^{\prime 2} \rightarrow 4$. One can show diagrammatically (Fig. 6) how the singularity type depends on the direction of motion in the $\left(\beta_{1}, \beta_{2}\right)$ plane when these equations for asymptotic behaviour hold. (Note that the restrictions on the motion implied by the allowed range of $\alpha$ in Bianchi type I reflect only an initial choice of numbering of axes.)

The behaviour when ${ }^{9} p=\mu$ is slightly different. At small $l, \mu \bumpeq M l^{-6}$, where $M$ is constant, say, and so

$$
\left.\begin{array}{rl}
3 l^{2} & \bumpeq\left(\Sigma^{2}+M\right) l^{-4} \Rightarrow l^{3} \bumpeq\left(3\left(\Sigma^{2}+M\right)\right)^{\frac{1}{2}} t \\
l_{v} & \simeq\left(3\left(\Sigma^{2}+M\right)\right)^{\frac{1}{6}} t^{\frac{1}{3}\left(1+\frac{2 \Sigma \sin \alpha_{v}}{\left(\Sigma^{2}+M\right)^{1 / 2}}\right) .}
\end{array}\right\}
$$

A pancake singularity is now impossible and if $M>3 \Sigma^{2}$ we must have a point singularity. For $3 \Sigma^{2} \geqq M>0$ it is convenient to introduce the parameter $\psi=\sin ^{-1}\left(\frac{\sqrt{\Sigma^{2}+M}}{2 \Sigma}\right)>\frac{\pi}{6}$. The possible singularity types are then as displayed in Table 2: all those shown can occur in Bianchi type I, while inspection of the table for $\alpha=0$ gives the type $\mathrm{V}$ possibilities (namely a cigar if $M<2 \Sigma^{2}$, barrel if $M=2 \Sigma^{2}$ and point if $M>2 \Sigma^{2}$ ).

${ }^{9}$ We strictly wish to ensure that $\mu w^{-2} \rightarrow 0$ as $\mu \rightarrow \infty$. This condition is violated if, for example, $p=\mu$ for some component of the fluid. For brevity we denote the alternatives by $\mu>p$ and $\mu=p$ in what follows. A sufficient condition for the former is $p \leqq(1-\varepsilon) \mu$ where $\varepsilon>0 . p>\mu$ violates causality. 
Table 2. Singularity types for (3.1) with $p=\mu$

\begin{tabular}{llll}
\hline & cigar & barrel & point \\
\hline$\psi=\pi / 2$ & none & $\alpha=\pi / 6$ & other values \\
$\pi / 2>\psi \geqq \pi / 3$ & $\psi-\pi / 3<\alpha<2 \pi / 3-\psi$ & $\alpha=2 \pi / 3-\psi$ & $\alpha<\psi-\pi / 3$ \\
& & and $\alpha=\psi-\pi / 3$ & and $\alpha>2 \pi / 3-\psi$ \\
$\pi / 3>\psi$ & $\alpha<2 \pi / 3-\psi$ & $\alpha=2 \pi / 3-\psi$ & $2 \pi / 3-\psi<\alpha<\pi / 3+\psi$ \\
& and $\alpha>\pi / 3+\psi$ & and $\pi / 3+\psi$ & \\
\hline
\end{tabular}

Thus all the singularity types introduced earlier occur for some Bianchi type I models, cf. [30]. The results given above and in Table 2 agree with the exact solutions for particular equations of state discussed by Jacobs [28]. In the next sections it is shown that the types of singularities so far encountered are not an exhaustive set [11, 13, 17, 31, 32].

Turning now to the question of the late stages of evolution one notes that if $\Lambda$ is non-zero it would dominate the $l$ equation at large $l$. Thus if $\Lambda<0$ there must be a maximum of $l$ followed by collapse to a second singularity. This second singularity will obey the same analysis as the first, save that the time direction is reversed and so the integral $\int \frac{d t}{l^{3}}$ changes sign. This implies that non-L.R.S. models have a second cigar singularity but with a different preferred axis and that L.R.S. cases that were initially pancakes (respectively, cigars) finally become cigars (respectively, pancakes).

If $\Lambda>0$ then, at large $l, l=\sqrt{\Lambda / 3} l$ so that

$$
l_{v} \bumpeq K_{v} l \exp \left(\frac{-2 \Sigma \sin \alpha_{v}}{3 \sqrt{\Lambda} l^{3}}\right)
$$

where the $K_{v}$ are constants. Hence the model becomes asymptotically Robertson-Walker in both senses defined earlier, for, at large $l, \sigma / \theta$ is $O\left(l^{-3}\right)$ while $\boldsymbol{\beta}$ is $\boldsymbol{\beta}_{0}+O\left(l^{-3}\right)$ where $\boldsymbol{\beta}_{0}$ is constant.

If $\Lambda=0$ the situation is a little more complicated. $E>0$ implies that $l \rightarrow \sqrt{\frac{10 E}{3}}$ as $l \rightarrow \infty$. In this case $\sigma / \theta$ is $O\left(l^{-2}\right)$ and $\boldsymbol{\beta}-\boldsymbol{\beta}_{0}$ is $O\left(l^{-2}\right):$ the models are again approximately Robertson-Walker in both the senses defined earlier. If $E=0$ the behaviour at large $l$ depends on the matter content. If ${ }^{10} p=\mu$ then $\mu=M l^{-6}, M$ constant, and $\sigma / \theta$ is constant (cf. [28]). If $p<\mu$ then $\sigma / \theta \rightarrow 0$ as $l \rightarrow \infty$. The stronger criterion given above is certainly satisfied if there is some $\gamma<2$ such that $\mu l^{3 \gamma}$ is bounded

\footnotetext{
${ }^{10}$ Strictly we must distinguish between cases where $\mu w^{-2} \rightarrow \infty$ as $\mu \rightarrow 0$ (" $p<\mu$ ") and where it does not $(p=\mu)$ (cf. note 9).
} 
below by a positive number. Since in the real universe it seems certain that $\mu l^{3} \rightarrow$ constant as $l \rightarrow \infty[6]$ we may take this condition to be satisfied.

Therefore we conclude that except in certain physically unlikely special cases all these models are asymptotically Robertson-Walker in both senses.

\section{The General Singularity Behaviour: Matter Dynamically Negligible}

In this and the two subsequent sections the cases covered by Section 3 are excluded. Thus we shall discuss only cases in which $V_{1}(\boldsymbol{\beta})$ and $\boldsymbol{\beta}$ are not constant. One may note that

i) in all cases the $\Lambda$ term is negligible near the singularity,

for (2.10) proves that $\Phi e^{4 \Omega}$ increases with $\Omega$ so that $\Phi e^{6 \Omega} / \Lambda \rightarrow \infty$ as $\Omega \rightarrow \infty$. Moreover as $\Phi$ and $V_{1}$ are non-negative (2.10) shows that $\Phi$ is monotone decreasing with $\Omega$. $\Phi$ is bounded below, therefore

ii) $\Phi$ tends to a finite non-negative limit as $\Omega \rightarrow \infty$.

Defining $\beta^{\prime}$ by $\beta^{\prime 2}:=\beta_{1}^{\prime 2}+\beta_{2}^{\prime 2}$, which is consistent with (2.3) we find from (2.8) that if $-R^{*} / 2+\mu+\Lambda$ is positive, $\left|\beta^{\prime}\right| \leqq 2$. In the exceptional case where $R^{*}$ may be positive (Bianchi type IX) this is still true sufficiently near the singularity, for even the least favourable admissible matter term $\left(p=0, \mu=M e^{3 \Omega}, M\right.$ constant) implies $\mu-R^{*} / 2+\Lambda>0$ for large $\Omega$. Thus

iii) $\left|\beta^{\prime}\right| \leqq 2$ sufficiently near the singularity.

For clarity we phrase the discussion of the next two sections in terms of the validity and consequences of the following three assumptions:

a) the $\Phi$-term dominates (2.8),

b) $V_{1}$ is small compared with $\beta^{\cdot 2} e^{-2 \Omega}$,

c) $\partial V / \partial \beta_{1}, \partial V / \partial \beta_{2}$ are small compared with $e^{-3 \Omega} \beta_{1}, e^{-3 \Omega} \beta_{2}$.

When a) is valid (2.8) implies

$$
2 \Omega \bumpeq-\sqrt{\Phi} e^{3 \Omega}
$$

and if in particular $\Phi \rightarrow C^{2}$, where $C>0$, as $\Omega \rightarrow \infty$ one may integrate (4.1) as

$$
l^{3} \bumpeq \frac{3 C}{2} t
$$

This volume evolution law is the same as for the early stages of the Bianchi I models discussed above (cf. [30]). It would remain true until the effects of the differences in (2.9) or (2.10) from the Bianchi I case, or the dynamical effect of the matter, became important. 
a) may fail to be valid in two ways which it is useful to separate. If $\Phi \rightarrow C^{2}>0$ as $\Omega \rightarrow \infty$, then the matter term can only be dynamically important at early times if ${ }^{9} p=\mu$. If instead $\Phi \rightarrow 0$ as $\Omega \rightarrow \infty$, then there would be equations of state less extreme (and more physically plausible) than $p=\mu$ such that matter would be dynamically important at early times. These possibilities are considered in the following section. Here we shall assume a) is valid and examine the self-consistency of this assumption, i.e. we examine only models in which near the singularity the matter has negligible effect on the evolution.

Finally concerning a) we note that it does not assert that the space is empty. The presence of some matter (although dynamically insignificant) is necessary to ensure that there is a real physical singularity (see e.g. $[30,33])$. However the fact that matter is dynamically negligible enables us to check our calculations by reference to the known exact vacuum solutions, which are summarised in [1].

b) implies that $\Phi$ is approximately constant and if a) is also true there is an epoch throughout which(4.2) holds and $\left|\beta^{\prime}\right| \bumpeq 2$. For the models we are considering $V_{1}(\boldsymbol{\beta}) \neq 0$ and so given $\Phi$ and $\Omega$ there will be certain regions of the $\boldsymbol{\beta}$-plane in which b) clearly fails. These are essentially of two sorts which we may describe as walls and valleys (see Figs. 1-5, 7-9).

c) implies that $\left(\beta_{1}{ }^{\circ}, \beta_{2}{ }^{\circ}\right) e^{-3 \Omega}$ is approximately constant. If a) and b) and c) are all true for $\Omega>\Omega_{0}, \Omega_{0}$ being constant, then for $\Omega>\Omega_{0}$

$$
\beta_{1}^{\prime} \bumpeq \text { constant }, \quad \beta_{2}^{\prime} \bumpeq \text { constant }, \quad \beta_{1}^{\prime 2}+\beta_{2}^{\prime 2} \bumpeq 4 .
$$

The asymptotic behaviour near the singularity if (4.3) holds is like that of the cases discussed in Section 3 and so can be simply read off from Fig. 6. c) fails in certain regions of the $\beta$ plane, for any given $\Phi$ and $\Omega$, and in general these regions are the same as where b) fails. The implications of b) and c) outlined above indicate clearly that it is the behaviour in these regions which determines the self-consistency of a) and therefore we now investigate this behaviour, no further consideration of models which never enter regions where b) and c) fail being required.

First let us consider the potential walls. In each case it is convenient to define new coordinates $\left(\beta_{\perp}, \beta_{\|}\right)$by rotation in the $\left(\beta_{1}, \beta_{2}\right)$ plane, so that the potentials at the walls take the form $V_{1} \bumpeq V_{0} e^{m \beta_{\perp}}$ where $V_{0}, m$ are constants. Such walls occur in the following cases: in the $n=0$ cases of Class B from (2.5), $m$ being $\frac{+2 k}{\sqrt{3+k^{2}}}$ and $\beta_{\|} \equiv 0$ : in Class A cases with $m=4$ and in the following regions (see Figs. 1-5); in type II at all points, in types $\mathrm{VI}_{0}$ and $\mathrm{VII}_{0}$ at large $\left|\beta_{2}\right|$, in types VII and IX at (for example) small $\beta_{2}$ and large positive $\beta_{1}$ : and in type $\mathrm{VI}_{0}$ with $m=2$ when $\beta_{2} \equiv 0$. 
The equations (assuming a) holds) are (4.1) and, by (2.9) and (2.10),

$$
\left.\begin{array}{l}
\Phi^{\prime} \bumpeq-4 V_{0} e^{m \beta_{\perp}-4 \Omega} \\
\left(\sqrt{\Phi} \beta_{\|}^{\prime}\right)^{\prime} \bumpeq 0, \\
\left(\sqrt{\Phi}\left(\beta_{\perp}^{\prime}-m\right)\right)^{\prime}=0 .
\end{array}\right\}
$$

Thus, up to small correction terms, $\sqrt{\Phi} \beta_{\|}^{\prime}$ and $\sqrt{\Phi}\left(m-\beta_{\perp}^{\prime}\right)$ are constant. (There are several vacuum cases in which (4.4) become exact and analytic solutions have been found: namely in type II [34], type III $(n=0)$ [4] and types $\mathrm{VI}_{0}(n=0)$ and $\mathrm{VI}_{h}(n=0)[1]$.)

The effective potential walls, represented by the value of $V_{1} e^{-4 \Omega}$, move outward, as $\Omega$ increases, at a speed $\beta_{\text {wall }}^{\prime}=4 / \mathrm{m}$. From ( $\left.4.4 \mathrm{a}\right)$ it is clear that $\beta_{1}^{\prime}$ must reduce below $4 / m$ in a finite time, for otherwise $\Phi$ becomes negative. The upper limit allowed for this time decreases with $\Omega$. If the approximations are valid indefinitely $V_{1} e^{-4 \Omega}$ decreases to zero, so b) will eventually hold (as will c).

The actual behaviour implied by these considerations depends on the value of $m$. In general in the Class A cases $m=4$ while $\left|\beta^{\prime}\right| \lesssim 2$. After the bounce $\beta_{L}^{\prime}<1$ and if $\mathrm{b}$ ) eventually holds this shows that the eventual state is one in which the system is either moving away from the wall, or towards it at an angle to the normal greater than $\pi / 3$. By calculation from the constants given above one can in fact construct a bounce law for the initial (i) and final ( $f$ ) angles between the motion and the normal $[11,17]$. These values are of course only asymptotically achieved at times when $V_{1} e^{-4 \Omega} \rightarrow 0$. Similarly one finds that (for $m=4$ )

$$
\Phi_{i}\left(5-2 \beta_{\perp}^{\prime}\right)_{i}=\Phi_{f}\left(5-2 \beta_{\perp}^{\prime}\right)_{f}
$$

showing that a lower limit for $\Phi_{f}$ is $\Phi_{i} / 9$.

In types $\mathrm{II}_{1} \mathrm{VI}_{0}$ and $\mathrm{VII}_{0}$ we now have all the information we require about bounces off walls. In type II there is just one bounce. In types $\mathrm{VI}_{0}$ and $\mathrm{VII}_{0}$ the walls at large $\left|\beta_{2}\right|$ are inclined to each other at an angle of $\pi / 3$ so that only a finite number of bounces occur before either the system escapes or it enters the valley region at small $\left|\beta_{2}\right|$ and large negative $\beta_{1}$. Thus if the valley reverses the motion the system eventually escapes to infinity. In the special type $\mathrm{VI}_{0}$ case with $\beta_{2} \equiv 0$ a wall with $m=2$ operates and, remembering that in this case $\beta_{\perp}^{\prime}<2$, reverses the motion. In all these cases $\Phi$ is only reduced by a non-zero factor by collisions with the walls, so that a) can be self-consistent. Moreover the results given here are consistent with the vacuum solutions for type II [34] and the special $(n=0)$ case of type $\mathrm{VI}_{0}$ in which $\beta_{2} \equiv 0[1]$.

In types VIII and IX there may be infinitely many collisions with the walls, and if so $\Phi \rightarrow 0$ as $\Omega \rightarrow \infty$. Misner has given an lower limit for this 
decrease, based on calculations using a square-well potential with the same bounce characteristics as the real potential and having walls which move at speed $\beta_{\text {wall }}^{\prime}=\frac{4}{m}=1$. For large $\Omega$ it is $\Phi \gtrsim K \Omega^{-2}$ where $K$ is constant $[11,17]$. This limit, which may clearly be carried over to the type VIII case, shows that collisons with walls could only affect the selfconsistency of a) if there is a very extreme equation of state.

In the $n=0$ cases of Class $\mathrm{B},|m|<2$. As $\Phi$ is positive and monotonedecreasing the constant $\sqrt{\Phi}\left(\beta_{\perp}^{\prime}-m\right)$ shows that either $\beta_{\perp}^{\prime}<m$ or $2 \geqq \beta_{\perp}^{\prime}>m$ or $\beta_{\perp}^{\prime}=m$ for all $\Omega$. If initially $m>\beta_{\perp}^{\prime}>0$ then a) and b) do not both hold, but the motion may reverse so that $\beta_{\perp}^{\prime} \simeq-2$. If $\beta_{\perp}^{\prime}>m$ initially then since $\Phi$ decreases $\beta_{\perp}^{\prime}$ must increase. In the marginal case $\beta^{\prime} \bumpeq m$, where small corrections are important, there is a possibility that $\Phi \rightarrow 0$ in such a way that a plausible matter content becomes dominant (or is at all times dominant); see next section. We may understand the perhaps unexpected behaviour of these cases by comparing them with the Class A cases whose $\beta_{\perp}$ motion is not reversed by collison with the wall. The essential difference arises from the constraint (2.2), cf. remarks in Section 2.

These results for the Class $\mathrm{B} n=0$ cases agree with the vacuum solutions for type III (L.R.S.) [4] and for type $\mathrm{VI}_{h}$ models of Case Bbi, the latter being (cf. [1])

$$
\left.\begin{array}{rl}
t & =\int_{0}^{u} X(p) d p ; X^{2}(u)=\left(\sinh 2 a_{0} u\right)^{1+k^{2}}\left(\tanh a_{0} u\right)^{k Q} \\
Y^{2} & =\left(\sinh 2 a_{0} u\right)^{1+k}\left(\tanh a_{0} u\right)^{Q} \\
Z^{2} & =\left(\sinh 2 a_{0} u\right)^{1-k}\left(\tanh a_{0} u\right)^{Q}
\end{array}\right\}
$$

where $Q= \pm \sqrt{3+k^{2}}$.

In the Bbii case (where $k=-3$ ) $V_{2}$ must be considered as a potential wall of a different kind from those above. Since $\Phi \geqq V_{2}>0$ we find there is an absolute upper limit to $\beta$ (cf. Fig. 9). For large $\Omega, V_{1}$ is negligible near this limit so that b) holds while c) fails. (2.9) then implies a rapid reversal of the $\beta$ motion. At very early times one would expect that $\beta^{\prime} \simeq 2$ just after this bounce and so a further reversal by the $V_{1}$ wall would not occur. However if when colliding with the $V_{2}$ wall, $\beta^{\prime}<m$ the possibility arises of more than one oscillation of $\beta$. It appears that potentials like $V_{2}$ may appear in those more complicated cases which this paper ignores, although a proper discussion requires more than a 2-dimensional $\beta$ space, cf. $[12,16]$.

Having considered the effects of potential walls we come now to the valleys. These occur in types $\mathrm{VI}_{0}, \mathrm{VII}_{0}$, VIII and IX at large negative $\beta_{1}$ and small $\left|\beta_{2}\right|$ (and in two further regions in type IX, see Fig. 5). There 
the potentials have effectively the following forms for motion in which $\beta_{2}$ 丰 0 :

Type $\mathrm{VI}_{0}$

$$
V_{1} \bumpeq \frac{4}{3} e^{-2 \beta_{1}}\left(1+3 \beta_{2}^{2}\right) .
$$

Types VII $_{0}$, VIII, IX

$$
V_{1} \bumpeq 4 \beta_{2}^{2} e^{-2 \beta_{1}} \text {. }
$$

Clearly if (4.8) reverses the $\beta_{1}$ motion, so will (4.7). We wish to consider motions in which initially $\beta_{1}^{\prime} \simeq-2$ and $\beta_{2}^{\prime} \neq 0$. We write $\beta_{1} \simeq-\beta_{0}-2 \Omega$ where $\beta_{0}$ is slowly varying. In first approximation $\beta_{2}$ executes simple harmonic motion with frequency $\omega=4 e^{\beta_{0}} / \sqrt{\Phi}$ and energy

$$
E=\frac{1}{2} \frac{\sqrt{\Phi}}{2}\left(\beta_{2}^{\prime 2}+\omega^{2} \beta_{2}^{2}\right),
$$

by (2.9). There is actually an exact solution of (2.9) when (4.8) holds, due to Chitre [17], in which the amplitude of the $\beta_{2}$ motion increases, so that (4.8) ceases to be valid, and the motion is no longer "down the valley". Alternatively this conclusion may be reached by consideration of the adiabatic invariant $K=E / \omega[11]$ and the secular changes of $\Phi$, and $\beta_{0}$ that can be deduced from it (Misner, unpublished). These considerations lead to the conclusion that the valley does in fact reverse the $\beta_{1}$ motion $^{11}$. A full proof would require some study of those regions where b) and c) fail but neither (4.4) nor (4.8) are valid approximations. However, it seems highly unlikely that this would provide any new effects.

The reflection by the valley does not entirely satisfy the obvious analogy of an electron in a magnetic bottle, because the energy is not conserved. To obtain an estimate of the rate of decrease of $\Phi$ we rewrite (2.10) as

$$
\left(\Phi e^{2 \Omega}\right)^{\prime}=2\left(\beta^{2} e^{-6 \Omega}-V_{1}(\boldsymbol{\beta}) e^{-4 \Omega}\right) e^{2 \Omega} .
$$

Now while (4.8) holds, $\left\langle\beta_{2}^{2} e^{-6 \Omega}\right\rangle \bumpeq\left\langle V_{1}(\boldsymbol{\beta}) e^{-4 \Omega}\right\rangle$, where \langle\rangle denotes time-average. Also $\left\langle\beta_{1}^{2} e^{-6 \Omega}\right\rangle>0$, so (4.10) implies that $\Phi e^{2 \Omega}$ increases. We see that a faster rate of decrease of $\Phi$ can occur than in collisons with the walls, and consideration of the diagrams (Figs. 2-5) shows that one would expect the fastest rate of decrease of $\Phi$ to occur in the valleys. Thus we conclude that a) is self-consistent if $\mu \geqq 3 p$. It should also be noted that the system may spend considerable periods in the valley regions while undergoing the reversal just discussed, with implications that will be discussed in Section 7.

Finally we must consider the cases that enter a valley region with $\beta_{2} \equiv 0$. These are L.R.S. spaces, except for the type $\mathrm{VI}_{0}$ case we have

11 This is borne out by selected numerical calculations due to Okerson [17] and Matzner et al. [43]. 
already discussed. The type $\mathrm{VII}_{0}$ L.R.S. case admits a type I group [1] and its possible asymptotic behaviours are the same (for here $\beta_{2}=0 \Rightarrow V_{1}$ $=0)$. In the remaining cases, L.R.S. spaces of types VIII and IX, the system can escape to infinity so as to produce a pancake singularity. (In type IX this follows because a) asserts that $\Phi e^{4 \Omega}>1$ ). This conclusion agrees with the behaviour of the known vacuum solutions $[34,35]$ and the numerical models of Behr [15].

We may combine the above results to reach the following conclusions concerning the behaviour of those models where a) holds, (these results being expressed in terms of the conventions of [1] and Table 1 regarding coordinates and structure constants). Note a) fails in Robertson-Walker universes (see Section 3).

Class A. In general the models have cigar singularities or are oscillatory; L.R.S. cases may have point or pancake singularities.

Type I: The motion of the system is rectilinear in the $\beta$-plane. The system escapes to infinity. The general case has a cigar singularity but some L.R.S. cases have pancake singularities (see Fig. 6).

Type II: The motion of the system is eventually at an angle of more than $\pi / 3$ to the ingoing normal to the wall, after a collision with the wall. In general a cigar singularity with the 2 or 3 axis distinguished. The L.R.S. case has a pancake singularity with the 1 axis distinguished. Type $V I_{0}$ : After possibly a few bounces on the walls and reversal by the "valley" of the $\beta_{1}$ motion, the system escapes to infinity in the $\boldsymbol{\beta}$-plane, in such a way (Fig. 6) as to give a cigar singularity on the 1 axis.

Type VII ${ }_{0}$ : If L.R.S., then the model is type I (q.v.); if non-L.R.S. the behaviour is as for type $\mathrm{VI}_{0}$.

Type VIII: If L.R.S., escapes along the $\beta_{1}$ axis and has a pancake singularity. Non-L.R.S. cases oscillate in the potential well, striking the walls and being reversed by the valley in general infinitely often (cf. [13]). Long periods may be spent in the valley.

Type IX: The motion again has in general an oscillatory character, spending much time in the valleys of the potential $[11,31]$. The L.R.S. cases bounce a few times along the axis and then escape to infinity, yielding a pancake solution [15].

It may be noted that in types VIII and IX it is the possibility of motion down the valley that prevents us drawing the conclusion that $(\Omega-|\beta|) \rightarrow \infty$, which if true would imply a point singularity.

Also we see that in type IX the bounces at early epochs occur at negative $R^{*}$. This means that a model with compact three-surfaces of homogeneity (i.e. of type IX, cf. $[1,2]$ ) will not in general have positive spatial curvature at all times. In cosmology "closed" meaning "having 
compact spatial three-surfaces of homogeneity", has been taken as synonymous with "having spatial three-surfaces of homogeneity of positive curvature". The latter two phrases are clearly inequivalent. (Also the space sections are always closed in the four dimensional topology.) The usage is misleading.

Class B with $\boldsymbol{n}=\mathbf{0}$. In general these cases have cigar singularities.

$B a$ : type $V$ : Cigar along 2 or 3 axis. The potential, being constant, has no effect.

Bbi: type III (L.R.S.): Motion only reversed by the potential if initially $1>\beta^{\prime}>0$. Cigar or pancake singularities can occur, and the exact solutions show this [4].

$B b i$ : type $V I_{h}$ : If $k=q / a<-1$ cigar along the 1 or 3 axis; if $-1<k<+1$ cigar along the 2 or 3 axis; if $k>1$ cigar along the 1 or 2 axis. In all cases the only motions that are reversed are those with $\beta^{\prime}$ initially between zero and $2 k / \sqrt{3+k^{2}}$.

Bbii: type $V I_{h}: k=-3$. Motion has $\beta$ bounded above. There is an extra time dependent function $f\left(x^{0}\right)$ in the metric, but this does not affect the singularity type which is a cigar with the 2 axis distinguished.

In all this we have assumed a) valid. Our calculations show that this is self-consistent in most cases. The possible exceptions are: in any model if ${ }^{9} p=\mu$; in types VIII and IX, if $\mu<3 p$; in certain Case Bb models. We have now to investigate what happens if a) fails.

\section{Special Cases: Matter Dynamically Important}

We shall examine what happens when the "anisotropy energy" $\Phi e^{6 \Omega}$ is not the dominant term in (2.8). Near the singularity this would mean that $\mu$ dominated, see remarks in Sections 1 and 4. There has been some controversy over the physically possible, and the appropriate, equations of state at high density [36]. One can argue that, although causality allows $\mu=p$, realistically one should impose the condition $\mu \geqq 3 p$. It is clear that matter with the extreme equation of state $p=\mu$ will in general cause a) to fail. Matter with $\mu \geqq 3 p$ need not do so in general. In this section we shall thus refer to the two representative cases $p=\mu\left(\mu=M e^{6 \Omega}\right)$ and $\mu=3 p\left(\mu=M e^{4 \Omega}\right)$, where $M$ is a constant.

If $p=\mu$ the approximation to (2.8) at large $\Omega$ is

We then find

$$
4 \Omega^{\cdot 2} \bumpeq\left(\frac{4}{3} M+\Phi\right) e^{6 \Omega} .
$$

$$
\left|\beta^{\prime}\right| \lesssim 2 /\left(1+\frac{4 M}{3 \Phi}\right)^{\frac{1}{2}}
$$


Thus if

$$
\begin{gathered}
4 M>9 \Phi, \\
\left|\beta^{\prime}\right|<1 .
\end{gathered}
$$

In a collision with a wall $(4.4 \mathrm{a})$ still holds, so that if $(4.3,4 \mathrm{a})$ are valid one finds that the $\beta_{\perp}$ motion need not be reversed. The equations that replace $(4.4 \mathrm{~b})$ show

$$
\left(\sqrt{\Phi+\frac{4 M}{3}}\right)\left(m-\beta_{\perp}^{\prime}\right) \text { and }\left(\sqrt{\Phi+\frac{4 M}{3}}\right) \beta_{\|}^{\prime}
$$

to be constant. The bounce law (4.5) holds when $m=4$, if we replace $\beta_{\perp}^{\prime}$ by $\beta_{\perp}^{\prime} /\left(1+\frac{4 M}{3 \Phi}\right)^{\frac{1}{2}}$ and $\Phi$ by $\Phi+\frac{4 M}{3}$. Thus a reversal of motion in collision with a wall is possible if

$$
2 /\left(1+\frac{4 M}{3 \Phi}\right)^{\frac{1}{2}} \gtrsim \beta_{\perp}^{\prime} \geqq \frac{4}{m}
$$

or, in Class B $(n=0)$ cases, when $0<\beta_{\perp}^{\prime}<m$. The final angle between the trajectory and the ingoing normal to the wall will not now have to be more than $\pi / 3$, but greater than some new critical angle less than $\pi / 3$.

Since the equation of state $p=\mu$ is physically implausible we shall not pursue the full details of the possibilities arising from it. We remark that (5.4) implies a point singularity in all cases, and that the condition (5.3) which led to it is precisely the generalisation of the condition $M>3 \Sigma^{2}$ for the cases of Section 3. When (5.3) does not hold at early times, as it may not in some models, the possible singularities will be as discussed in Section 4, with the addition of the barrel case (cf. Table 2).

If $\Phi \rightarrow 0$ as $\Omega \rightarrow \infty$, and ${ }^{9} p<\mu$ then a) must hold. So if $p<\mu$ and a) fails, $\Phi \rightarrow 0$. Thus $\beta^{\cdot 2} e^{-6 \Omega} \rightarrow 0$ and this requires that for every $\Omega_{0}$ such that $\beta^{*} \neq 0$, there is some $\Omega>\Omega_{0}$ at which the potential term in (2.9) opposes the velocity in the $\beta$ plane. Therefore it is clear for example that if in type II $\beta_{\perp}^{\prime}<0$ a) eventually holds.

If the right-hand side of $(2.9)$ is not significant then $\left(\beta_{1}{ }^{\circ}, \beta_{2}{ }^{\circ}\right) e^{-3 \Omega}$ $\bumpeq$ constant (i.e. $c$ ) holds) and so a) would eventually hold provided $\mu \geqq 3 p$. Now the regions in which the right-hand side of (2.9) are significant move outwards in the $\beta$-plane at speeds $\beta_{c}^{\prime}$ of order 1. If $\Phi e^{6 \Omega}$ is small compared with $\mu$, then $\left|\beta^{\prime}\right|<<1$ and so the system cannot enter, or if in, cannot remain in, regions where b) and/or c) fail. Therefore the system would evolve so that a) eventually held. The conclusion is that it is not in general self-consistent for a) to be false at all times. The problem we are left with is determining what happens when a) is also not self-consistent.

Since we are now assuming ${ }^{9} p<\mu$, the relevant cases are Bianchi types VIII and IX, and $n=0$ cases of the subclass Bb. In the two Class A 
cases the rate of decrease of $\Phi$ when a) holds is such that $\Phi e^{2 \Omega}$ increases, so that matter with equation of state such that $\mu \geqq 3 p$ would be negligible at all sufficiently early times. For intermediate equations of state $\mu>p>\mu / 3$ one would expect that there will in general be alternating epochs in which $\mu$ and $\Phi e^{6 \Omega}$ are the dominant terms in (2.8); clearly there will also be at least some equations of state with $p<\mu$ for which the matter is never negligible dynamically.

In the Class B cases the exceptional cases occur when, during the period in which $\Phi$ dominates, $\beta^{\prime}=m$ (see Section 4). There is an exact solution due to Kantowski [4] which demonstrates that in this case one can in fact have a point singularity. It is an L.R.S. model of Bianchi type III $(n=0)$ filled with disordered radiation $\left(p=\frac{1}{3} \mu\right)$ in which

$$
\left.\begin{array}{l}
Z=a\left(1-\left(\frac{t-t_{0}}{a}\right)^{-\frac{2}{3}}\right)^{\frac{1}{2}}, \\
X=a\left(\left(\frac{t-t_{0}}{a}\right)^{\frac{2}{3}}-1\right)^{\frac{1}{2}}\left(\frac{t-t_{0}}{a}\right)^{\frac{2}{3}} .
\end{array}\right\}
$$

At large $t, \beta^{\prime}=1=m$ and $\Phi$ dominates in (2.8). As $\Omega \rightarrow \infty\left(t \rightarrow a+t_{0}\right)$ $\Phi$ becomes negligible compared with the matter content. To show that models of the same kind as (5.6) occur in the other Class B $n=0$ cases could only be proved by giving explicitly the exact solutions, but it seems highly likely.

\section{Behaviour Far from the Singularity}

To discuss the behaviour far from the initial singularity it is helpful to introduce the variables

$$
\chi=-\Omega ; \quad \psi=\Phi e^{4 \Omega}=\beta^{2} e^{2 \chi}+V(\boldsymbol{\beta})=\frac{4}{3} \sigma^{2} e^{2 \chi}+V_{1}(\boldsymbol{\beta}) .
$$

The governing equations are (2.9) and

$$
\begin{aligned}
& \frac{d \psi}{d \chi}=-4\left(\beta^{\cdot 2} e^{2 \chi}+V_{2} e^{-4 \chi}\right)=-\frac{16}{3} \sigma^{2} e^{2 \chi}, \\
& 4 \chi^{2}=\frac{4}{3}(\mu+\Lambda)+\psi e^{-2 \chi}-\delta_{I X}^{T} e^{-2 \chi},
\end{aligned}
$$

where $T$ is the group type. We wish to consider the behaviour when $\chi \rightarrow \infty$ (for models other than those of Section 3). The energy density $\mu$ obeys $M e^{-6 \chi} \leqq \mu \leqq M e^{-3 \chi}$ where $M$ is an initial value (assuming $0 \leqq p \leqq \mu$ ). Since it seems that in the present-day real universe $\mu \simeq M e^{-3 x}$ [6] we shall assume this in what follows (suitable amendment of the argument and conclusions could be made for other cases). 
(6.1) shows that $\psi$ is monotone decreasing with $\chi$ and since it is nonnegative, it therefore tends to a finite limit as $\chi \rightarrow \infty$. The existence of this limit does not however enable us to conclude from (6.1) that $\sigma^{2} e^{2 \chi} \rightarrow 0$ as $\chi \rightarrow \infty$.

Clearly if $\Lambda \neq 0$ the $\Lambda$ term will dominate (6.2) at large $\chi$. If $\Lambda>0$ the universe will expand indefinitely except in certain cases of Bianchi type IX. At large $\chi, \chi^{\cdot 2} \bumpeq \Lambda / 3$ and as $\left(\beta^{\cdot} e^{\chi}\right)^{2}$ is bounded above by the initial value of $\psi$, say $\psi_{0}$, we find that $|\beta / \chi|$ is bounded above by $\sqrt{\frac{3}{\Lambda}} \psi_{0} e^{-\chi}$. This tends to zero as $\chi \rightarrow \infty$, and thus $\sigma / \theta \rightarrow 0$. Moreover if the approximation for $\chi^{\cdot}$ is valid for $\chi>\chi_{0}$

$$
\left|\int_{\chi_{0}}^{\infty} \beta \cdot d t\right|<\sqrt{\frac{3}{\Lambda}} \psi_{0} \int_{\chi_{0}}^{\infty} e^{-\chi} d \chi=\sqrt{\frac{3}{\Lambda}} \psi_{0} e^{-\chi_{0}} .
$$

(6.3) shows that $\boldsymbol{\beta}$ tends to some finite value. The models with $\Lambda>0$ become approximately Robertson-Walker in both the senses defined in Section 3, except for certain cases of Bianchi type IX which collapse to a second singularity.

The Bianchi IX models may collapse to a second singularity because $R^{*}$ can be positive. The critical value $\Lambda_{c}$ of $\Lambda$ required to prevent this in an anisotropic model is not larger than the value required for the corresponding Robertson-Walker model (i.e. the well-known $\Lambda_{c}$ of Lemaitre models), since the terms appearing in (6.2) in the anisotropic case which vanish in the Robertson-Walker case are strictly non-negative. Moreover, there may be borderline cases in which $\chi$ asymptotically approaches a fixed $\chi_{0}$ from below (as in certain Robertson-Walker cases [6]).

If $\Lambda<0$ the universe must reach a maximum of $\chi$ (i.e. of $l$ ) and then collapse to a second singularity. The behaviour near this singularity obeys the analysis of Sections 4 and 5. If only one kind of behaviour is admissible for a certain type of model, the initial and final singularities must both exhibit this behaviour, but if more than one kind of behaviour is possible the two singularities may, and in certain cases must, be different. The details, which we omit, can easily be deduced from the work of Sections 3-5.

The case $\Lambda=0$ is more complicated, since it is not immediately clear which term in (6.2) will be dominant (unlike the cases of Section 3 where $V_{1}$ was constant, and so $\psi$ became dominant at large $\chi$ if $V_{1} \neq 0$ ). Let us suppose that $\psi$ dominantes (so that $\psi e^{\chi}$ is large at large $\chi$ ) and consider first the Class A cases other than type IX. When $\psi$ dominates the arguments of Section 4, time-reversed, apply. Thus $\psi e^{2 x}$ is on average decreasing with time even in the type VIII case, and hence $\psi e^{x} \rightarrow 0$. Our

12 Except in Bianchi types VIII and IX, the arguments and qualitative conclusions of this section are easily generalised from the dust case to the radiation case. 
assumption is not self-consistent. This proves that $\psi e^{j x} \rightarrow 0$ for all $j<1$ and in particular $\psi \rightarrow 0$. Now $\psi \rightarrow 0$ implies $V_{1} \rightarrow 0$ and so in types II, $\mathrm{VI}_{0}$ and VIII $|\beta| \rightarrow \infty$. Moreover, that $\psi e^{j x} \rightarrow 0$ for $j<1$ gives a finite lower limit in these cases for the time average of $d \beta / d \chi$. Hence there is no time $\chi_{0}$ such that $\psi$ is negligible in (6.2) for all $\chi>\chi_{0}$. For these Bianchi types (II, $\mathrm{VI}_{0}$, VIII) we conclude that matter and anisotropy are both dynamically important at late stages in these models, and that these models do not become asymptotically Robertson-Walker in either of the senses defined earlier. In Bianchi type $\mathrm{VII}_{0}$ it is not clear what happens since $V \rightarrow 0$ if the model asymptotically lies on the $\beta_{1}$ axis. In this case the system approximates to a Bianchi I L.R.S. model and both the criteria of becoming asymptotically Robertson-Walker could be satisfied.

In the Bianchi IX case a similar argument shows that $(\psi-1) e^{x} \rightarrow 0$ if $(\psi-1) e^{-2 x}$ is the dominant term in (6.2). Thus as $\psi \rightarrow 1$ there arises a stage at which matter is dynamically important. If eventually $\psi<1$ we can see that $(\psi-1) e^{-2 \chi}<0$ would dominate (6.2) at large $\chi$ and so that the model must reach a maximum of $\chi$ and then collapse to a second singularity (which would be like the first in general behaviour). Although there are strong arguments for thinking that this happens, cf. [43], the present author has not found a rigorous proof.

In the Class B $(n=0)$ models the time reversal of the arguments of Section 4 shows that $\sqrt{\Phi}\left(\frac{d \beta}{d \chi}+m\right)$ is constant if $\psi$ dominates (6.2). Since then $\Phi=\psi e^{4 \chi} \rightarrow \infty$ the approximation is only self-consistent if $\frac{d \beta}{d \chi} \rightarrow-m$. In this case we find that asymptotically $V e^{\chi} \sim O\left(e^{\left(1-m^{2}\right) \chi}\right)$ so that $V e^{\chi} \rightarrow 0$ if $m^{2}>1$. Thus there can be a self consistent solution of this kind only when $m^{2} \leqq 1$ (i.e. $|q| \leqq|a|$ ). Such solutions do in fact occur, for the L.R.S. type III $(n=0)$ models of Kantowski and Sachs [4] contain several exact solutions of this kind. Among these are all their general dust and radiation solutions with $\Lambda=0$, including (5.6). These certainly do not satisfy either of the criteria of "becoming Robertson-Walker".

When in Class B $(n=0) \frac{d \beta}{d \chi} \rightarrow-m$ as $\chi \rightarrow \infty$ the assumption that $\psi$ dominates (6.2) cannot be self-consistent at all times and we may again conclude that $|\beta| \rightarrow \infty$ as $\chi \rightarrow \infty$ and that anisotropy and matter terms are both dynamically important at some late stages.

We may summarise the results obtained for models in which $\Lambda=0$ by remarking that among those considered the only group types in which models can become approximately Robertson-Walker are those which admit exactly Robertson-Walker models ${ }^{13}$.

13 This may be the underlying reason that Carswell could find no realistic L.R.S. model of a Bianchi type other than I, [25]. 


\section{Discussion}

The results of the previous sections have a number of implications for the models, considered as possible universes. We discuss these first, and then review the more general question of the validity of the models.

Briefly, the results are that the commonest type of singularity behaviour is the cigar singularity, while the two most complicated Bianchi types studied show oscillatory behaviour. Special cases can have pancake, point or barrel singularities (Sections 3-5). In Section 6 it is shown that those models with $\Lambda=0$ and realistic matter content which do not collapse to a second singularity (i.e. those not of type IX) cannot become asymptotically Robertson-Walker unless they belong to a Bianchi type to which a Robertson-Walker model also belongs.

The singularity behaviour affects the horizon structure. One motive for interest in this is that if cosmological models exist in which there are no particle horizons, then one may be able to account for the observed degree of homogeneity in the universe by physical processes rather than a priori symmetries $[6,10]$. Lack of horizons is also a help in accounting for present-day isotropy using dissipative processes in a homogeneous model $[10,11]$.

The topology of the covering spaces of the three-surfaces of homogeneity in our models is $R^{3}$, except for type IX where the space-sections are compact and the covering space is $S^{3}$ [2]. Of course identifications may be made in many cases without destroying the global isometry group, and in rather more cases without destroying local isometries.

Let us consider the Bianchi I models. In general there is a particle horizon. Hawking [37] pointed out that when there is a pancake singularity there is no horizon on the axis of symmetry (pancake cases must be L.R.S.). The metric is

$$
d s^{2}=-d t^{2}+t^{2}\left(d x^{1}\right)^{2}+\left(d x^{2}\right)^{2}+\left(d x^{3}\right)^{2}
$$

near the singularity. For an observer at the origin at time $t$, the furthest visible points are at

$$
\begin{aligned}
& x^{1}=\int_{0}^{t} \frac{\pi_{1} d t}{t^{2}\left(t^{-2} \pi_{1}{ }^{2}+\pi_{2}{ }^{2}+\pi_{3}{ }^{2}\right)^{\frac{1}{2}}} ; \\
& x^{2}=\int_{0}^{t} \frac{\pi_{2} d t}{\left(t^{-2} \pi_{1}{ }^{2}+\pi_{2}{ }^{2}+\pi_{3}{ }^{2}\right)^{\frac{1}{2}}} \\
& x^{3}=\int_{0}^{t} \frac{\pi_{3} d t}{\left(t^{-2} \pi_{1}^{2}+\pi_{2}{ }^{2}+\pi_{3}{ }^{2}\right)^{\frac{1}{2}}}
\end{aligned}
$$

where $\pi_{1} \pi_{2} \pi_{3}$ are constants (cf. [3]). Thus the observer can see everything in an infinite circular cylinder whose base, has radius $t$. (This slightly 
extends Hawking's statement.) Since the behaviour of geodesics along the principal axes in Class A models depends on the length scales in the same way as in Bianchi type I, we see that only pancake-like behaviour during the evolution will remove horizons, and that then either such behaviour must persist for infinite time or the universe must be of finite extent in the direction of the distinguished axis of the pancake. One expects that qualitatively similar conclusions apply to the Class B cases, and this is borne out by the analytic formulae available for the only case with $n=0$ where a pancake is possible, i.e. Bianchi type III (cf. $[3,38]$ ).

Thus to remove particle horizons completely we need a model in which pancake-like epochs occur and in which each of the three principal axes in turn is the distinguished axis. The only possible case is that of type IX ${ }^{14}$. (Note that the horizon may also be removed for one axis only in types I, VII 0 , VIII and III.) To prove that all horizons are removed in a general type IX universe we need an ergodic theorem applicable under the conditions of type IX evolution. (Standard ergodic theorems in mechanics assume constant energy, while here we have a timedependent energy.) It seems plausible that such a theorem exists, but the present author is unable to offer a proof. If the motion is not ergodic then it is possible the horizon may only be removed for one or two axes (leaving respectively a tube or slab of matter visible). To understand the importance of this we can calculate the amount of matter visible by comparison with a Bianchi I universe with identifications made at unit coordinate intervals so that the spatial sections have $T^{3}$ topology. If in this case the horizon is removed for one (respectively two) direction(s) the amount of matter visible at the time $t$ when the horizon is removed is $\pi \mu t^{3}$ (respectively $\pi \mu t^{2}$ ). Thus in terms of baryon number, whose density $\varphi$ behaves as $\varphi=M / l^{3}$ this amount is $\pi M t^{2}$ (respectively $\pi M t$ ) and is therefore insignificantly small at early times.

It is of interest to note that the removal of horizons at early stages is related to the topology of the space sections just as it is when considering the removal of horizons at late stages in a Lemaitre $(\Lambda>0)$ universe.

The second significance of the singularity behaviour is its effect on the helium production in the big bang. Hawking and Tayler [39] pointed out that in the Bianchi I models anisotropy markedly altered the timescale near the singularity (in fact to (4.2)) and the helium abundance could thus be drastically reduced. The helium production is dependent on the volume expansion alone [22] unless there are serious departures

${ }^{14}$ Doroshkevich and Novikov find that even if in this case all three horizons are removed the time-scales are such that the induced "mixing" is ineffective in reducing anisotropy [44]. 
from the local thermal equilibrium assumed in the calculations ${ }^{15}$. The amount of helium produced in various models has been calculated by a number of authors $[22,25,41]$. From Sections 4 and 5 we see that except in a few special cases (4.1) holds near the singularity in the models considered and that in most cases (4.2) also holds. Therefore we may expect that the dependence of the helium production in our models on the anisotropy and matter parameters is generally similar to that found by Thorne [22]. Only numerical calculation would show if this expectation is borne out in detail.

The qualitative behaviour of the evolution of the length scales for different directions is helpful in predicting that of the redshift-magnitude relation [3]. The general rule is that if the length scale in a particular direction increases slower (respectively, faster) than the average length scale, redshifts of objects in that direction appear lower (respectively, higher) than average ${ }^{16}$. Thus if for example there is a direction for which the length scale tends to a finite number as we approach the singularity, there will be a finite maximum redshift for objects in that direction. Therefore in type IX we expect to see oscillatory deviations from the Hubble Law while in type II we see one reversal of the deviations (when $\beta_{1}$ reverses). Using the expressions of [3] for the $r_{A}-z$ relations, and the above equations for the dynamics of the evolution, one could plot numerically the magnitude-redshift relation for any axis, and the dependence of the black-body temperature on angle, for any particular model. Some work of this kind has been carried out by Saunders [27] but he unfortunately overlooked the possibilities of reversal of motion in the $\beta$ plane.

Numerical computations of specific models of our class are of value in several ways. First we can determine whether there are models which do not become asymptotically Robertson-Walker (as found in Section 6) but in which there is a certain time when the model is observationally admissible. Secondly there are a number of points mentioned above about which it would be useful to have quantitative information, for example, one might want to find the detailed time-scales of the model, e.g. that for which (4.2) is valid, in terms of the model's physical parameters (e.g. matter density) see e.g. [44]. In certain cases (namely those of types VIII and IX) there is a difficulty in that the model performs infinitely many oscillations near the singularity and so one cannot use a computer to follow the evolution right back to the singularity. (One can of course use a new "regularised" time coordinate $\tau(t)$ such that $\tau(0)$

\footnotetext{
15 Strictly there must be some departure from thermal equilibrium in an expanding universe [40].

16 For fuller discussion of the behaviour of null geodesics in the models see [3]. 


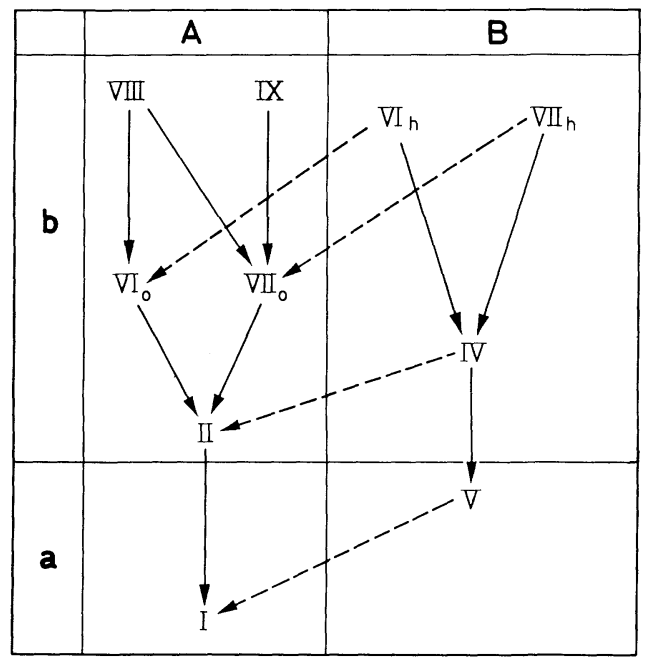

Fig. 10. Diagram showing specialisation of group types which can be obtained on letting non-zero parameters go to zero. A broken arrow changes the group class, an unbroken arrow does not

is infinite, e.g. $\tau \equiv \Omega$, in order to compute any given finite number of oscillations: cf. work by Okerson reported in [17], and [43].

The motivation for this paper and its predecessors has been to understand the dynamical effects of anisotropy of shear by carrying out an analysis of the simplest cases, i.e. anisotropic spatially-homogeneous cosmological models, basing this analysis on a canonical classification of their symmetry groups. Within the class of models considered it can be said that certain types of model are the most general, in a sense derived from Fig. 10. This shows how specialisation of group type occurs on setting certain parameters to zero (which of course does not represent a dynamic evolution). From this the most general cases, to which future studies should pay particular attention, are those of types VIII, IX, $\mathrm{VI}_{h}$ and $\mathrm{VII}_{h}$.

The models can be generalised by the introduction of a stress-energy tensor other than that for perfect fluid, or by no longer taking the fluid flow vector to be orthogonal to the surfaces of homogeneity, or both. A number of papers on these lines have appeared (e.g. [10, 16, 19, 22, 25, 28]). The introduction of anisotropic stress is especially interesting because it could counteract the mechanisms which cause certain models not to be asymptotically Robertson-Walker. If so, a rather wider class of models would become observationally admissible than if anisotropic stress were disregarded. Indeed certain types of anisotropic stress (e.g. 
those due to collisionless neutrinos or magnetic fields) in fact must act in this way (cf. forthcoming work by Jacobs and Hughston). There is controversy about the effectiveness of the dissipative processes, $[10,20$, $42,44]$ especially as it appears that in certain cases the fluid approximations usually made need not be valid at any epoch [42].

Acknowledgements. The author wishes to thank the following for their help; for stimulating discussions Drs. F. P. Bretherton, J. M. Stewart and B. G. Schmidt, Professor C. W. Misner, and especially Dr. S. W. Hawking; for informative communications Professor C. W. Misner and Dr. K. C. Jacobs; for a maintenance grant the United Kingdom Science Research Council; and above all his supervisor Dr. G. F. R. Ellis for constant stimulus, encouragement and help.

\section{References}

1. Ellis, G. F. R., MacCallum, M. A. H.: Commun. Math. Phys. 12, 108 (1969).

2. Schmidt, B. G.: Commun. Math. Phys. 15, 329 (1969).

3. MacCallum, M. A. H., Ellis, G. F. R.: Commun. Math. Phys. 19, 31 (1970).

4. Kantowski, R., Sachs, R. K.: J. Math. Phys. 7, 443 (1966); Kantowski, R.: Ph.D. thesis, University of Texas 1966.

5. Hawking, S. W., Ellis, G. F. R.: Phys. Letters 17, 246 (1965).

6. Ellis, G. F. R.: Lectures at the International School of Physics, Corso XLVII, Varenna, to be published (1969).

7. Hawking, S. W., Ellis, G. F. R.: Astrophys. J. 152, 25 (1968); Hawking, S. W., Penrose, R.: Proc. Roy. Soc. (London) A 314, 529 (1970).

8. Bianchi, L.: Lezioni sulla teoria dei gruppi continui finiti transformazioni. Pisa: Editiozi Spoerri 1918.

9. Estabrook, F. B., Wahlquist, H. D., Behr, C. G.: J. Math. Phys. 9, 497 (1968).

10. Misner, C. W.: Astrophys. J. 151, 431 (1968).

11. - Phys. Rev. Letters 22, 1071 (1969): Phys. Rev. 186, 1319 (1969).

12. Hawking, S. W.: Monthly Notices Roy. Astron. Soc. 142, 129 (1969).

13. Lifschitz, E. M., Khalatnikov, I. M.: Z.E.T.F. Pisma 11, 200 (1970), in Russian.

14. Gödel, K.: Proc. Int. Congr. Math. (Cambridge), Vol. I, 175 (1950).

15. Behr, C. G.: Astron. Abh. Hamburg. Sternwarte 7, 5 (1965); Z. Astrophys. 54, 268 (1962), and 60, 286 (1965).

16. Ozsvath, I.: University of Texas preprint "Spatially Homogeneous World Models" (1970).

17. Misner, C. W.: in Relativity, ed. M. Carmeli, S. Fickler and L. Witten. New York: Plenum Press 1970.

18. Ellis, G. F. R.: J. Math. Phys. 8, 1171 (1967); Stewart, J. M., Ellis, G. F. R.: J. Math. Phys. 9, 1072 (1968).

19. MacCallum, M. A. H., Stewart, J. M., Schmidt, B. G.: Commun. Math. Phys. 17, 343 (1970).

20. Misner, C. W.: Phys. Rev. 186, 1328 (1969).

21. ter Haar, D.: Elements of Hamiltonian mechanics. Amsterdam: North-Holland 1961.

22. Thorne, K. S.: Astrophys. J. 148, 51 (1967).

23. Rindler, W.: Monthly Notices Roy. Astron. Soc. 116, 662 (1956).

24. Penrose, R.: Battelle rencontres 1967, ed. C. M. de Witt and J. A. Wheeler. New York: Benjamin 1968.

25. Carswell, R.: Monthly Notices Roy. Astron. Soc. 144, 279 (1969); Ph. D. thesis, University of Sussex, 1969. 
26. Novikov, I. D.: Sov. Astron.-A.J. 12, 427 (1969); Russian original, Astron. Zh. 45, 538 (1968).

27. Saunders, P. T.: Ph.D. thesis, King's College, London (1967); Monthly Notices Roy. Astron. Soc. 142, 213 (1969).

28. Jacobs, K. C.: Astrophys. J. 153, 661 (1968) and 155, 379 (1969).

29. Kasner, E.: Am. J. Math. 43, 217 (1921).

30. Lifschitz, E. M., Khalatnikov, I. M.: Advanc. Phys. 12, 185 (1963).

31. Belinsky, V. A., Khalatnikov, I. M.: J.E.T.P. 29, 911 (1969) and to appear; Russian originals. Z.E.T.F. 56, $1700(1969)$ and 57, 2163 (1969).

32. Khalatnikov, I. M., Lifschitz, E. M.: Phys. Rev. Letters 24, 76 (1970).

33. Misner, C. W., Taub, A. H.: J.E.T.P. 28, 22 (1969); Russian original Z.E.T.F. 55, 233 (1968).

34. Taub, A. H.: Ann. Math. 53, 472 (1951).

35. Newman, E. T., Tamburino, L., Unti, T.: J. Math. Phys. 4, 915 (1963).

36. Harrison, E. R.: Astrophys. J. 142, 1643 (1965); Chanmugam, G.: J. Phys. A 3, L.19 (1970).

37. Hawking, S. W.: Adams Prize Essay, Cambridge University 1966.

38. Tomita, K.: Prog. Theor. Phys. 40, 264 (1968).

39. Hawking, S. W., Tayler, R. J.: Nature 209, 1278 (1966).

40. Ehlers, J.: Lectures at the International School of Physics, Varenna, Corso XLVII; to be published (1969).

41. Wagoner, R. V., Fowler, W. A., Hoyle, F.: Astrophys. J. 148, 3 (1967). Tayler, R. J.: Nature 217, 433 (1968).

42. Stewart, J. M.: Ph.D. thesis, Cambridge University (1969); Monthly Notices Roy. Astron. Soc. 145, 347 (1969).

43. Matzner, R. A., Shepley, L. C., Warren, D. R.: Ann. Phys. (N. Y.) 57, 401 (1970).

44. Doroshkevich, A. G., Novikov, I. D.: Preprint No. 15, Institute of Applied Mathematics, Acad. Sci. U.S.S.R., Moscow (1970).

45. Matzner, R. A.: Astrophys. J. 157, 1085 (1969).

46. Taub, A. H.: In: Proceedings of the 1967 Colloque sur fluides et champ gravitationnel. Paris: Centre National de la Recherches Scientifiques 1969.

\author{
M. A. H. MacCallum \\ Department of Applied Mathematics \\ and Theoretical Physics \\ Silver Street \\ Cambridge/England
}

